\title{
About Underappreciated Yet Active Conformations of Thiourea Organocatalysts
}

\author{
Adriana Supady, ${ }^{\dagger}$ Stefan Hecht, ${ }^{\ddagger}$ and Carsten Baldauf* ${ }^{\dagger}{ }^{\dagger}$ \\ ${ }^{\dagger}$ Fritz-Haber-Institut der Max-Planck-Gesellschaft, 14195 Berlin, Germany \\ ${ }^{\ddagger}$ Department of Chemistry, Humboldt-Universität zu Berlin, 12489 Berlin, Germany
}

\section{Supporting Information}

ABSTRACT: Conformational dynamics can define the function of organocatalysts. While the accepted mechanism of Schreiner's catalyst features a double hydrogen bond to the substrate that only forms with the anti-anti conformation of its central thiourea group, our electronic-structure theory study reveals that binding of the model substrate methyl vinyl ketone prefers syn-anti conformations. We find a new mechanism featuring $\pi$ stacking interactions and highlight the need for extensive structure searches for flexible molecules, especially when aiming for structure-based design of catalytic activity.

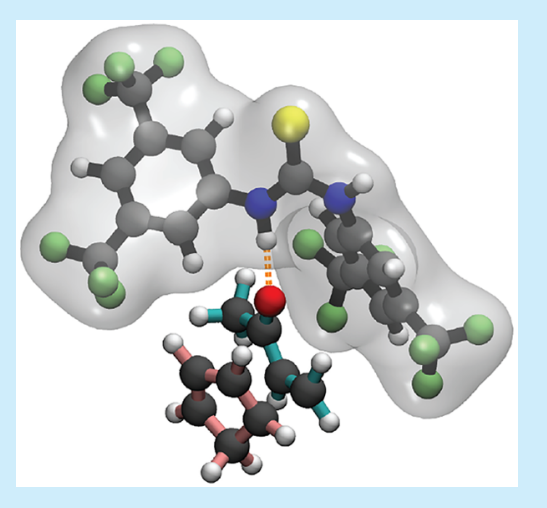

atalysts speed up reactions by binding substrates and by stabilizing transition states, thereby lowering reaction barriers. Noncovalent organocatalysts represent a highly selective and sustainable design alternative to conventional metal-based catalysts, ${ }^{1-4}$ e.g., by utilizing a combination of hydrogen bonding with entropic and enthalpic effects to accelerate reactions. An example is illustrated in Figure 1a: Schreiner's catalyst 1 catalyzes the Diels-Alder reaction of the dienophile methyl vinyl ketone (2) with cyclopentadiene (3) to the endo product 4. ${ }^{1}$ The established mechanism exploits the capability of $\mathrm{NH}$ protons in the anti-anti conformation of the central thiourea moiety to form a double hydrogen bond to the keto group of 2 (Figure $1 \mathrm{~b}$ ).

Organocatalysts such as $\mathbf{1}$ are flexible compounds with rich structural dynamics. Similarly to enzymes, ${ }^{3}$ their function is directly linked to their structure and their activity may be regulated via their conformational dynamics. Consequently, it is crucial to investigate their full conformational space when studying such flexible molecules. We performed first-principles structure searches ${ }^{5}$ at the dispersion-corrected ${ }^{6} \mathrm{PBE}^{7}$ level of density-functional theory with the FHI-aims code. ${ }^{8}$ All structures were rerelaxed using PBE augmented by Grimme's D3 dispersion correction ${ }^{9}$ in dichloromethane modeled by the COSMO approach $^{10}$ as implemented in ORCA. ${ }^{11}$ Gibbs energies were obtained by numeric rigid-rotor and harmonicoscillator calculations using D3 dispersion-corrected PBE and single-point $\mathrm{PBE0}^{12}$ calculations, both in COSMO dichloromethane. Dunning's correlation-consistent cc-pVTZ basis set was used. ${ }^{13}$ Our choice of functionals was influenced by good experience in testing and using PBE and PBE0 for biomolecules. $^{14-16}$ In the Supporting Information (SI), we demonstrate the agreement between the dispersion corrected
PBE and PBE0 functionals with coupled-cluster calculations for conformers of $\mathbf{1}$ and also the consistency among different functionals for the reaction we describe.

The global minimum structure is associated with the syn-syn conformation of the thiourea moiety, followed by the $3.1 \mathrm{kcal}$ $\mathrm{mol}^{-1}$ less stable syn-anti conformer (Figure 1c). The anti-anti conformation, capable of forming the double hydrogen bond depicted in Figure 1b, is energetically the least favored and 3.5 $\mathrm{kcal} \mathrm{mol}^{-1}$ less stable than the syn-syn conformer. Conformational changes between the minima involve rotations of the thiourea $\mathrm{N}-\mathrm{C}$ bonds as well as of the substituted phenyl groups and are hindered by conformational locking due to attractive interactions of the phenyl ortho-hydrogens with the sulfur atom. However, the resulting barriers in the range of 10 $\mathrm{kcal} \mathrm{mol}^{-1}$ are low enough to be overcome at room temperature.

Along with catalyst $\mathbf{1}$, the conformational preferences of three further thiourea based molecules were investigated (details can be found in the SI) among which the bifunctional asymmetric Takemoto catalyst ${ }^{17}$ is of particular interest. Interestingly, the syn-anti conformation is most stable for the Takemoto catalyst, while the anti-anti conformation is again energetically substantially penalized.

Environmental effects influence the conformational preferences of flexible bioorganic molecules. In crystals of $\mathbf{1}$, intermolecular double-hydrogen bonds lead to a preference for the anti-anti orientation of the central thiourea moiety. ${ }^{2}$ Our first-principles simulations in isolation as well as in dichloromethane (modeled as continuum), however, show that the syn-

Received: June 13, 2017

Published: August 8, 2017 


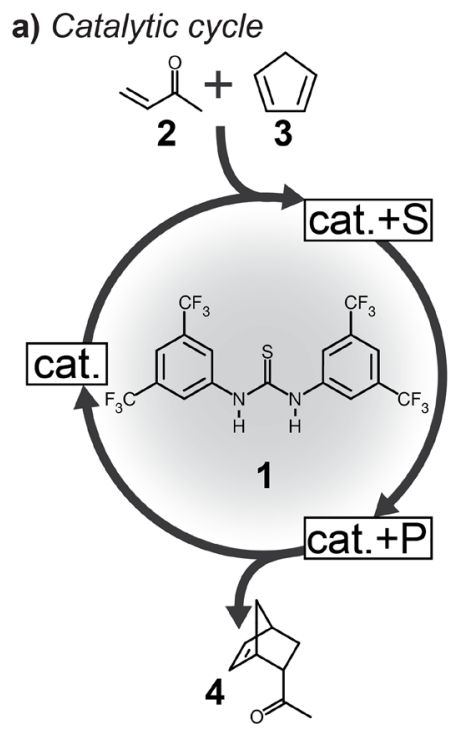

\section{b) Double hydrogen bond}

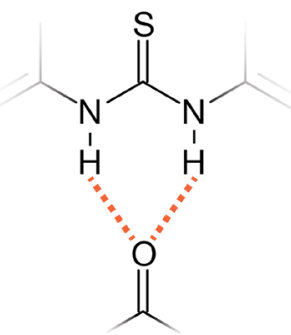

\section{c) Conformational flexibility of Schreiner's catalyst 1}
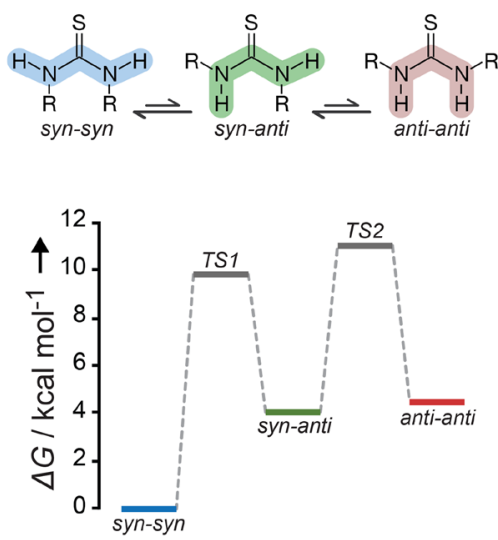

Figure 1. (a) Prototypical Diels-Alder reaction of the dienophile methyl vinyl ketone $\mathbf{2}$ with cyclopentadiene 3, catalyzed by Schreiner's catalyst $\mathbf{1}$, leads to the endo product 4. (b) Formation of a double hydrogen bond is the established catalytic mechanism of thiourea catalysts. (c) Plot showing relative Gibbs energies at $298.15 \mathrm{~K}$ in dichloromethane (PBE+D3/PBE0+D3) of minima and bond-rotation transition states of 1 .

syn conformer of 1 dominates. In agreement with our results, gas-phase free-energy calculations by others predict the syn-anti conformer of 1 to be more stable than the anti-anti conformer. $^{18-20}$ However, predictions for the syn-syn conformer deviate: It is predicted to be of similar stability as antianti in a study that relies on first-principles calculations without corrections for long-range dispersion. ${ }^{18}$ Another study employs the M06 functional, which includes dispersion by parametrization, but the syn-syn form of $\mathbf{1}$ is not considered. ${ }^{19}$ As in our study, Madarász et al. ${ }^{20}$ computed Gibbs energies by combining dispersion-corrected density-functional approximations with continuum simulations of an apolar solvent. Both studies independently predict the syn-syn conformation as most stable in solution. Clearly, long-range dispersion interactions have a big impact as it can be seen from the conformational energy hierarchies of $\mathbf{1}$ with and without Grimme's D3 dispersion correction compared to basis-set extrapolated coupled-cluster calculations (see the SI).

Also specific interactions, for example, with the substrates of the reaction, can affect conformational preferences. Hence, we investigated whether binding of Diels-Alder substrates $\mathbf{2}$ and $\mathbf{3}$ alters the population of different conformers of $\mathbf{1}$. Besides the anti-anti, syn-anti, and syn-syn conformers of the catalyst alone, we estimated Boltzmann populations at $298.15 \mathrm{~K}$ based on Gibbs energies also for the complexes $\mathbf{1}+\mathbf{2}$ and $\mathbf{1}+\mathbf{2}+\mathbf{3}$ in endo arrangements. In the case of the syn-anti conformer of $\mathbf{1}$, two different complexes are possible with either the substrates located on the opposite side of the sulfur atom and parallel to the phenyl ring of $\mathbf{1}$ (discussed in the following) or with the substrates located next to the sulfur atom of $\mathbf{1}$. The latter has been studied but is not discussed due to its unfavorable energetics.

Figure 2 summarizes the results: At room temperature, the Boltzmann population of 1 alone is dominated by the syn-syn conformer. Binding of dienophile 2 alters the share of catalyst conformations, and syn-anti is now most populated. Upon additional binding of 3 , essentially the formation of the catalyst-substrates complex (cat. $+S$ ) directly prior to the reaction barrier, syn-anti is dominating the population with a

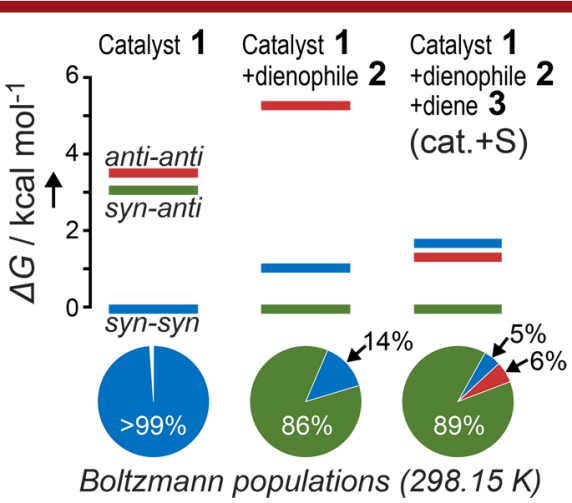

Figure 2. Relative Gibbs energies of conformers of Schreiner's catalyst 1 alone, bound to the dienophile methyl vinyl ketone 2 , and bound to 2 and cyclopentadiene 3 in dichloromethane (PBE+D3/PBE0+D3). Pie charts represent Boltzmann populations at $298.15 \mathrm{~K}$.

share of $89 \%$. The cat. $+\mathrm{S}$ complex that is based on the antianti conformer of 1 makes up only about $6 \%$ of the population.

We just found out that the anti-anti conformer of 1 , which is believed to be the catalytically active one, is very little populated. Consequently, we wanted to challenge the interpretation of this being the only catalytic mechanism of Schreiner's catalyst for such a Diels-Alder reaction. Based on minimum structures for the cat. $+\mathrm{S}(\mathbf{1}+\mathbf{2}+\mathbf{3})$ and catalystproduct complex (cat. $+\mathrm{P}, \mathbf{1}+\mathbf{4}$ ) states for the three conformer types syn-syn, syn-anti, and anti-anti of 1 , transitionpath searches were performed using the growing string method $^{21}$ as implemented in aimsChain. ${ }^{22}$ The resulting structures that are close to transition states (TS) were further optimized by the eigenvector following algorithm as implemented in Orca and verified by identifying exactly one imaginary frequency. The reaction profiles cat. $+\mathrm{S} \rightarrow \mathrm{TS} \rightarrow$ cat. $+\mathrm{P}$ are shown in Figure $3 \mathrm{a}$. The transition-state geometries in Figure $3 \mathrm{~b}$ give an impression of the mode of action of the different catalyst conformations. The anti-anti conformer of 1 involves dienophile $\mathbf{2}$ in a double hydrogen bond. The syn-anti conformer of 1 induces the formation of a $\pi$ sandwich of substrates and catalyst and aligns them for the reaction. The 


\section{a) Reaction profile}

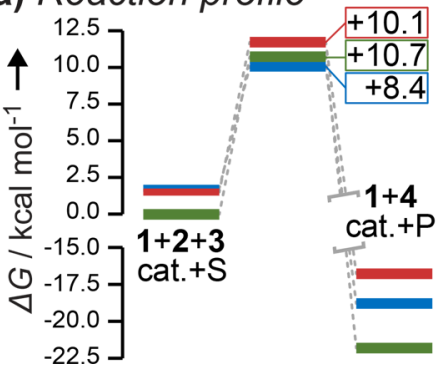

b) Transition-state geometries

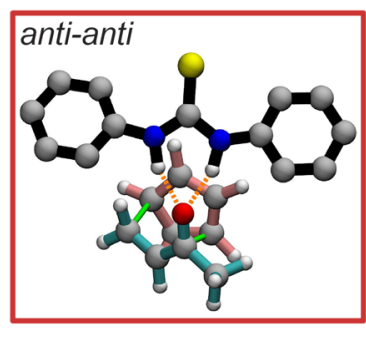

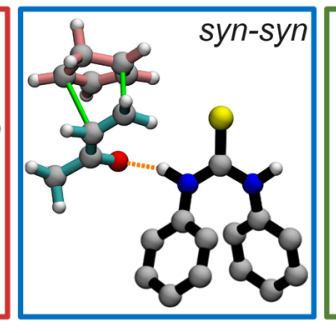

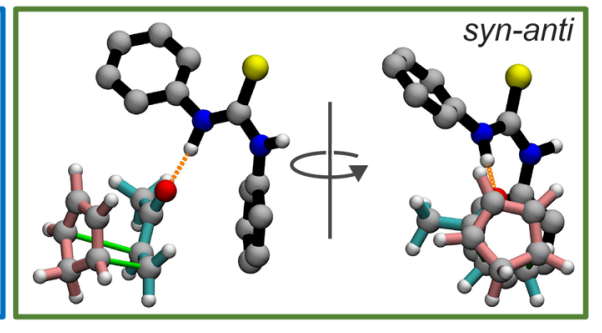

Figure 3. (a) Energy profiles of the Diels-Alder reaction of $\mathbf{2}$ and 3 catalyzed by different conformers of 1: anti-anti (red), syn-syn (blue), and synanti (green). The energy scale shows relative Gibbs energies $\Delta G$ at $298.15 \mathrm{~K}$ in dichloromethane (PBE+D3/PBE0+D3); relative barrier heights $\Delta \Delta G$ are shown next to the TS. (b) Structural representations of the transition states: trifluoromethyl groups and apolar hydrogens of 1 were omitted for clarity. Hydrogen bonds are highlighted in orange; coloring of covalent bonds indicates reaction partners (black, 1; red, 2; cyan, 3). Bonds to be formed during the reaction are highlighted in green. The TS related to the syn-anti conformer of $\mathbf{1}$ is shown in front and side view.

correct orientation of $\mathbf{2}$ is ensured by a single hydrogen bond. The syn-syn conformer of $\mathbf{1}$ leaves the substrates hanging freely and also only forms a single hydrogen bond.

The reaction barrier of the Diels-Alder reaction $2+3 \rightarrow 4$ without catalyst amounts to $12.3 \mathrm{kcal} \mathrm{mol}^{-1}$. The lowest absolute reaction barriers of 10.7 and $10.1 \mathrm{kcal} \mathrm{mol}^{-1}$, respectively, result from either the $\pi$ sandwich mechanism related to the syn-anti conformation of $\mathbf{1}$ or the freely hanging orientation of the substrates facilitated by the syn-syn conformer. The double hydrogen bond mechanism linked to the anti-anti conformation gives rise to an absolute barrier of $11.7 \mathrm{kcal} \mathrm{mol}^{-1}$ cat. $+\mathrm{S}$. The lowest barrier relative to the energy of the corresponding cat. $+\mathrm{S}$ complex is induced by the syn-syn conformer of $\mathbf{1}$ and amounts to $8.4 \mathrm{kcal} \mathrm{mol}^{-1}$. In all cases, the LUMO energy of the $\mathbf{1}+\mathbf{2}$ complexes is lowered in comparison to 2 alone (see the SI).

The different cat. $+\mathrm{S}$ complexes, characterized by the conformation of Schreiner's catalyst, represent a super basin of the energy landscape, most populated is the cat. $+S$ complex with 1 in a syn-anti conformation. The relative barriers $(\Delta \Delta G)$ of both the syn-syn and the commonly accepted anti-anti are lower than the one associated with the syn-anti conformer. In order to estimate which is the dominant mechanism, we define flux as the product of the reaction rate and the probability for the cat. $+S$ complex being in one of the possible states. The latter is known from the populations displayed in Figure 2. But how about the reaction rates out of the respective basins? According to harmonic transition-state theory (HTST), ${ }^{23}$ the reaction rate $k_{\text {HTST }}$ is a product of a prefactor and the Boltzmann probability based on the activation energy. The prefactor, which can be interpreted as an attempt frequency, does not vary significantly for the alternative paths (see the SI). But while the probability of overcoming the barrier (linked to the relative barrier height) is comparable for the double hydrogen bond mechanism (anti-anti) and the $\pi$ sandwich path (syn-anti), it is higher for the reaction path associated with the syn-syn conformer of $\mathbf{1}$, which is reflected in the reaction rates discussed in the SI. If we compare, for example, the $\pi$-stacking mechanism (syn-anti) with the double hydrogen bond mechanism (anti-anti), the latter is associated with an almost 10 times higher reaction rate while the former is more than 10 times higher populated.

Computer simulations represent approximations to reality that can cause errors. By comparing different functionals for describing the catalyzed chemical reaction (see Table S4 of the SI), we illustrate how the employed level of electronic-structure theory may eventually alter the findings. Furthermore, the use of an implicit solvation model as well as the rigid-rotor and harmonic-oscillator approximation for the Gibbs energies are possible sources of errors, ${ }^{24-26}$ a possible remedy for these shortcomings are first-principles molecular dynamics simulations ${ }^{27}$ with explicit solvent molecules. Given these approximations that we have to make, the least we can say is (i) Schreiner's catalyst $\mathbf{1}$ is a flexible molecule that can adopt multiple conformations, (ii) the anti-anti conformation that is linked to the established double hydrogen bond mechanism is energetically penalized and scarcely populated, and (iii) instead, we find alternative reaction mechanisms: the syn-syn conformation of the catalyst binds 2 with a single hydrogen bond, while syn-anti involves aromatic interactions ${ }^{28}$ to align substrates and catalyst in a $\pi$ sandwich that exhibits some resemblance of an enzyme's binding pocket; monomethylated variants of 1 represent a potential way of proving this proposed mechanism. Our computational studies have provided detailed mechanistic insights into the field of thiourea catalysts; the emergence of two synergistic reaction mechanisms might explain their robustness, e.g., for protic as well as for aprotic solvents. We open new routes for the in silico design of thiourea catalysts, but we also highlight the importance of carefully elucidating conformational space in the field of organocatalysis in general.

\section{ASSOCIATED CONTENT}

\section{Supporting Information}

The Supporting Information is available free of charge on the ACS Publications website at DOI: 10.1021/acs.orglett.7b01782.

Computational methodology and further relevant results as referred to in the text (PDF)

Structures in $x y z$ format and simulation output files (ZIP)

\section{AUTHOR INFORMATION}

\section{Corresponding Author}

*E-mail: baldauf@fhi-berlin.mpg.de. ORCID ${ }^{\circ}$

Stefan Hecht: 0000-0002-6124-0222 Carsten Baldauf: 0000-0003-2637-6009 Notes

The authors declare no competing financial interest. 


\section{ACKNOWLEDGMENTS}

The authors thank Luca Ghiringhelli (FHI) for discussions and Matthias Scheffler (FHI) for continuous support.

\section{REFERENCES}

(1) Wittkopp, A.; Schreiner, P. R. Chem. - Eur. J. 2003, 9, 407-414.

(2) Schreiner, P. R. Chem. Soc. Rev. 2003, 32, 289-296.

(3) Yoon, T. P.; Jacobsen, E. N. Science 2003, 299, 1691-1693.

(4) Taylor, M. S.; Jacobsen, E. N. Angew. Chem., Int. Ed. 2006, 45, $1520-1543$.

(5) Supady, A.; Blum, V.; Baldauf, C. J. Chem. Inf. Model. 2015, 55, $2338-2348$.

(6) Tkatchenko, A.; Scheffler, M. Phys. Rev. Lett. 2009, 102, 073005.

(7) Perdew, J. P.; Burke, K.; Ernzerhof, M. Phys. Rev. Lett. 1996, 77, $3865-3868$.

(8) Blum, V.; Gehrke, R.; Hanke, F.; Havu, P.; Havu, V.; Ren, X.; Reuter, K.; Scheffler, M. Comput. Phys. Commun. 2009, 180, 21752196.

(9) Grimme, S.; Antony, J.; Ehrlich, S.; Krieg, H. J. Chem. Phys. 2010, 132, 154104.

(10) Klamt, A.; Schüürmann, G. J. Chem. Soc., Perkin Trans. 2 1993, 799.

(11) Neese, F. WIREs: Comput. Mol. Sci. 2012, 2, 73-78.

(12) Adamo, C.; Barone, V. J. Chem. Phys. 1999, 110, 6158.

(13) Dunning, T. H. J. Chem. Phys. 1989, 90, 1007.

(14) Schubert, F.; Pagel, K.; Rossi, M.; Warnke, S.; Salwiczek, M.; Koksch, B.; von Helden, G.; Blum, V.; Baldauf, C.; Scheffler, M. Phys. Chem. Chem. Phys. 2015, 17, 5376-5385.

(15) Schubert, F.; Rossi, M.; Baldauf, C.; Pagel, K.; Warnke, S.; von Helden, G.; Filsinger, F.; Kupser, P.; Meijer, G.; Salwiczek, M.; Koksch, B.; Scheffler, M.; Blum, V. Phys. Chem. Chem. Phys. 2015, 17, 7373-7385.

(16) Marianski, M.; Supady, A.; Ingram, T.; Schneider, M.; Baldauf, C. J. Chem. Theory Comput. 2016, 12, 6157-6168.

(17) Okino, T.; Hoashi, Y.; Takemoto, Y. J. Am. Chem. Soc. 2003, $125,12672-12673$.

(18) Kirsten, M.; Rehbein, J.; Hiersemann, M.; Strassner, T. J. Org. Chem. 2007, 72, 4001-4011.

(19) Lippert, K. M.; Hof, K.; Gerbig, D.; Ley, D.; Hausmann, H.; Guenther, S.; Schreiner, P. R. Eur. J. Org. Chem. 2012, 2012, 59195927.

(20) Madarász, A.; Dósa, Z.; Varga, S.; Soós, T.; Csámpai, A.; Pápai, I. ACS Catal. 2016, 6, 4379-4387.

(21) Peters, B.; Heyden, A.; Bell, A. T.; Chakraborty, A. J. Chem. Phys. 2004, 120, 7877-7886.

(22) Yao, Y.; Supady, A.; Baldauf, C.; Scheffler, M.; Ghiringhelli, L. M. Manuscript in preparation.

(23) Vineyard, G. H. J. Phys. Chem. Solids 1957, 3, 121-127.

(24) Grimme, S. Chem. - Eur. J. 2012, 18, 9955-9964.

(25) Cheng, G.-J.; Zhang, X.; Chung, L. W.; Xu, L.; Wu, Y.-D. J. Am. Chem. Soc. 2015, 137, 1706-1725.

(26) Jensen, J. H. Phys. Chem. Chem. Phys. 2015, 17, 12441-12451.

(27) Soto-Delgado, J.; Tapia, R. A.; Torras, J. J. Chem. Theory Comput. 2016, 12, 4735-4742.

(28) Krenske, E. H.; Houk, K. N. Acc. Chem. Res. 2013, 46, 979-989. 